Annals of Pure and Applied Mathematics

Vol. 17, No. 1, 2018, 123-134

ISSN: 2279-087X (P), 2279-0888(online)

Published on 12 May 2018

Annals of

www.researchmathsci.org

DOI: http://dx.doi.org/10.22457/apam.v17n1al4

Pure and Applied

Mathematics

\title{
Fuzzy Semi-Open Sets and Fuzzy Pre-Open Sets in Fuzzy Quad Topological Space
}

\author{
Ranu Sharma ${ }^{1}$, Bhagyashri A. Deole ${ }^{2}$ and Smita Verma ${ }^{3}$ \\ Department of Applied Mathematics and Computational Science \\ SGSITS, Indore (M.P.), India \\ Email: ${ }^{2}$ deolebhagyashri@gmail.com, ${ }^{3}$ yvsmita@gmail.com \\ Corresponding author. Email: ranusamarth@gmail.com
}

Received 14 March 2018; accepted 30 April 2018

Abstract. The aim of this paper is to introduce two new types of fuzzy open sets namely fuzzy q-semi-open sets and fuzzy q-pre-open sets in fuzzy q-topological spaces and also defined the fuzzy continuity namely fuzzy q-continuity, fuzzy q-semi-continuity and fuzzy q-pre-continuity in fuzzy q-topological spaces.

Keywords: fuzzy q-semi-open sets, fuzzy q-pre-open sets, fuzzy q-continuity, fuzzy qsemi-continuity and fuzzy q-pre-continuity.

\section{AMS Mathematics Subject Classification (2010): 54A40}

\section{Introduction}

Levine [11] introduced the idea of semi-open sets and semi-continuity in topological space and Mashhour et al. [3] introduced the concept of pre-open sets and pre continuity in a topological space. Maheshwari and Prasad [14] introduced semi-open sets in bitopological spaces. Jelic [8] generalized the idea of pre-open sets and pre continuity in bitopological space.

The study of tri-topological space was first initiated by Kovar [9]. Palaniammal [15] studied tri topological space and introduced semi and pre-open sets in tri topological space and he also introduced fuzzy tri topological space. Hameed and Moh. Abid [10] gives the definition of 123 open set in tri topological spaces. We [17] studied properties of tri semi-open sets and tri pre-open sets in tri topological space. Mukundan [5] introduced the concept on topological structures with four topologies, quad topology) and defined new types of open (closed) set. We have [18] introduced semi and pre-open sets in quad topological spaces.

In 1965, Zadeh [7] introduced the concept of fuzzy sets. In 1968 Change [4] introduced the concept of fuzzy topological spaces. Kandil [1] introduced fuzzy bitopological spaces in 1991, Fuzzy semi-open sets and fuzzy semi continuous mappings 
in fuzzy topological spaces was studied by Azad [6]. Bin [2] defined the concept of preopen sets in fuzzy topological space. Thakur and Malviya [16] introduced semi-open sets, semi continuity in fuzzy bitopological spaces. Sampath Kumar [13] defined a $\left(\tau_{i}, \tau_{j}\right)$ fuzzy pre-open set and characterized a fuzzy pair wise pre continuous mappings on a fuzzy bitopological space. We have [12] introducedfuzzy tri semi-open sets and fuzzy tri pre-open sets, fuzzy tri continuous function, fuzzy tri semi-continuous function and fuzzy tri pre-continuous functions and their basic properties.

In this paper, we introduce fuzzy q-semi-open sets, fuzzy q-pre-open sets, fuzzy qcontinuity, fuzzy q-semi-continuity and fuzzy q-pre-continuity and their fundamental properties in fuzzy q-topological space.

\section{Preliminaries}

Definition 2.1. [12] Consider two fuzzy tri topological spaces $\left(X, \tau_{1}, \tau_{2}, \tau_{3}\right)$, $\left(Y, \tau_{1}^{\prime}, \tau_{2}^{\prime}, \tau_{3}^{\prime}\right)$. A fuzzy function $f: I^{X} \rightarrow I^{Y}$ is called a fuzzy tri continuous function if $\chi_{\lambda}$ is fuzzy tri open in $X$, for every tri open set $\chi_{\lambda}$ in $\mathrm{Y}$.

Definition 2.2. [12] Let $\left(X, \tau_{1}, \tau_{2}, \tau_{3}\right)$ be a fuzzy tri topological space then a fuzzy subset $\chi_{\lambda}$ of $X$ is said to be fuzzy tri semi-open set if $\chi_{\lambda} \leq \operatorname{cl}\left(\right.$ int $\left.\chi_{\lambda}\right)$ and complement of fuzzy tri semi-open set is fuzzy tri semi-closed. The collection of all fuzzy tri semi-open sets of $X$ is denoted by $\operatorname{tri}-F S O(X)$

Definition 2.3. [12] Let $\left(X, \tau_{1}, \tau_{2}, \tau_{3}\right)$ be a fuzzy tri topological space then a fuzzy subset $\chi_{\lambda}$ of $X$ is said to be fuzzy tri pre-open set if $\chi_{\lambda} \leq t r i-i n t\left(t r i-c l \chi_{\lambda}\right)$ and complement of fuzzy tri pre-open set is fuzzy tri pre-closed. The collection of all fuzzy tri semi-open sets of $X$ is denoted by $\operatorname{tri}-F P O(X)$.

Definition 2.4. [5] Let $X$ be a nonempty set and $\tau_{1}, \tau_{2}, \tau_{3}$ and $\tau_{4}$ are general topologies on $X$.Then a subset $A$ of space $X$ is said to be quad-open(q-open) set if $A \prec \tau_{1} \vee \tau_{2} \vee \tau_{3} \vee \tau_{4}$ and its complement is said to be q-closed and set $X$ with four topologies called q-topological spaces $\left(X, \tau_{1}, \tau_{2}, \tau_{3}, \tau_{4}\right)$.

Definition 2.5. [5] Let $\left(X, \tau_{1}, \tau_{2}, \tau_{3}, \tau_{4}\right)$ be a quad topological space and let $A \subset X$ . The intersection of all q-closed sets containing $\mathrm{A}$ is called the q-closure of A \& denoted by $q-c l A$.We will denote the q-interior (resp. q-closure) of any subset, say of $\mathrm{A}$ by $q-\operatorname{int} A(q-c l A)$, where $q-c l A$ is the union of all q-open sets contained in $\mathrm{A}$, and $q-c l A$ is the intersection of all q-closed sets containing A. 
Fuzzy Semi-Open Sets and Fuzzy Pre-Open Sets in Fuzzy Quad Topological Space

3. Fuzzy q-semi-open sets and fuzzy q-pre-open sets in fuzzy q-topological space

Definition 3.1. Let $\mathrm{X}$ be a nonempty set $\tau_{1}, \tau_{2}, \tau_{3}$ and $\tau_{4}$ are fuzzy topologies on $\mathrm{X}$. Then a fuzzy subset $\chi_{\lambda}$ of space $\mathrm{X}$ is said to be fuzzy q-open if $\chi_{\lambda} \prec \tau_{1} \cup \tau_{2} \cup \tau_{3} \cup \tau_{4}$ and its complement is said to be fuzzy q-closed and set $\mathrm{X}$ with four fuzzy topologies called fuzzy q-topological spaces $\left(X, \tau_{1}, \tau_{2}, \tau_{3}, \tau_{4}\right)$.

Definition 3.2. Let $\left(X, \tau_{1}, \tau_{2}, \tau_{3}, \tau_{4}\right)$ be a fuzzy quad topological space and let $\chi_{\lambda} \prec X$ The intersection of all fuzzy q-closed sets containing $\chi_{\lambda}$ is called the fuzzy q-closure of $\chi_{\lambda}$ and denoted by $\operatorname{Fqcl}\left(\chi_{\lambda}\right)$. We will denote the fuzzy q-interior (resp. fuzzy qclosure) of any fuzzy subset, say of $\chi_{\lambda}$ by fuzzy $F q \operatorname{int}\left(\chi_{\lambda}\right)\left(\operatorname{Fq} \operatorname{cl}\left(\chi_{\lambda}\right)\right)$, where $F q \operatorname{int}\left(\chi_{\lambda}\right)$ is the union of all fuzzy q-open sets contained in $\chi_{\lambda}$, and $\operatorname{Fqcl}\left(\chi_{\lambda}\right)$ is the intersection of all fuzzy q-closed sets containing $\chi_{\lambda}$.

Definition 3.3. Let $\left(X, \tau_{1}, \tau_{2}, \tau_{3}, \tau_{4}\right)$ be a fuzzy q-topological space then a fuzzy subset $\chi_{\lambda}$ of $X$ is said to be fuzzy q-semi-open set if

$$
\chi_{\lambda} \leq \operatorname{Fqcl}\left(\text { Fqint } \chi_{\lambda}\right)
$$

Complement of fuzzy q-semi-open set is called fuzzy q-semi-closed set. The collection of all fuzzy q-semi-open sets of $X$ are denoted by $\mathrm{F} q S O(X)$

Example 3.4. Let $X=\{a, b, c, d\}$ be a non-empty fuzzy set.

Consider four fuzzy topologies on $\mathrm{X}$

$$
\begin{aligned}
\tau_{1} & =\left\{\tilde{1} X, \tilde{0} X, \chi_{\{a\}}\right\}, \\
\tau_{2} & =\left\{\tilde{1} X, \tilde{0} X, \chi_{\{a, d\}}\right\}, \\
\tau_{3} & =\left\{\tilde{1} X, \tilde{0} X, \chi_{\{c, d\}}\right\}, \\
\tau_{4} & =\left\{\tilde{1} X, \tilde{0} X, \chi_{\{a, c, d\}}\right\} .
\end{aligned}
$$

Fuzzy open sets in fuzzy q-topological spaces are union of all four fuzzy topologies.

Fuzzy q-open sets of

$$
X=\left\{\tilde{1} X, \tilde{0} X, \chi_{\{a\}}, \quad \chi_{\{a, d\}}, \chi_{\{c, d\}}, \quad \chi_{\{a, c, d\}}\right\} .
$$

Fuzzy q-semi-open sets of $X$ are denoted by

$$
F q S O(X)=\left\{\tilde{1} X, \tilde{0} X, \chi_{\{a\}}, \chi_{\{a, d\}}, \chi_{\{c,, d\}}, \chi_{\{a, c, d\}}\right\}
$$


Definition 3.5. Let $\left(X, \tau_{1}, \tau_{2}, \tau_{3}, \tau_{4}\right)$ be a fuzzy q-topological space then a fuzzy subset $\chi_{\lambda}$ of $X$ is said to be fuzzy q-pre-open set if $\chi_{\lambda} \leq$ Fqint $\left(F q c l \chi_{\lambda}\right)$. Complement of fuzzy q-pre-open set is called fuzzy q-pre-closed set. The collection of all fuzzy q-preopen sets of $X$ is denoted by $\mathrm{F} q P O(X)$.

Example 3.6. Let $X=\{a, b, c, d\}$ be a non-empty fuzzy set.

Consider four fuzzy topologies on $\mathrm{X}$

$$
\begin{aligned}
\tau_{1} & =\left\{\tilde{1} X, \tilde{0} X, \chi_{\{a\}}\right\}, \\
\tau_{2} & =\left\{\tilde{1} X, \tilde{0} X, \chi_{\{a, d\}}\right\}, \\
\tau_{3} & =\left\{\tilde{1} X, \tilde{0} X, \chi_{\{b, d\}}\right\}, \\
\tau_{4} & =\left\{\tilde{1} X, \tilde{0} X, \chi_{\{a, c, d\}}\right\} .
\end{aligned}
$$

Fuzzy open sets in fuzzy q-topological space are union of all four fuzzy topologies. Then fuzzy q-open sets of

$$
X=\left\{\tilde{1} X, \tilde{0} X, \chi_{\{a\}}, \chi_{\{a, d\}}, \chi_{\{b, d\}}, \chi_{\{a, c, d\}}\right\} .
$$

Fuzzy q-pre-open sets of $X$ denoted by

$$
F q S O(X)=\left\{\tilde{1} X, \tilde{0} X, \chi_{\{a\}}, \chi_{\{a, d\}}, \chi_{\{b, d\}}, \chi_{\{a, c, d\}}\right\} .
$$

Definition 3.7. Let $\left(X, \tau_{1}, \tau_{2}, \tau_{3}, \tau_{4}\right)$ be a fuzzy q-topological space. The intersection of all fuzzy q-semi-closed sets of $X$ containing a fuzzy subset $\chi_{\lambda}$ of $X$ is called fuzzy q-semi closure of $\chi_{\lambda}$ and is denoted by $\operatorname{F} q \operatorname{sint}\left(\chi_{\lambda}\right)$.

Definition 3.8. Let $\left(X, \tau_{1}, \tau_{2}, \tau_{3}, \tau_{4}\right)$ be a fuzzy q-topological space. The intersection of all fuzzy q-pre-closed sets of $X$ containing a fuzzy subset $\chi_{\lambda}$ of $X$ is called fuzzy q-pre closure of $\chi_{\lambda}$ and is denoted by Fqpint $\left(\chi_{\lambda}\right)$.

Definition 3.9. Let $\left(X, \tau_{1}, \tau_{2}, \tau_{3}, \tau_{4}\right)$ be a fuzzy q-topological space. The intersection of all fuzzy q-semi-closed sets of $X$ containing a fuzzy subset $\chi_{\lambda}$ of $X$ is called fuzzy q-semi closure of $\chi_{\lambda}$ and is denoted by Fqscl $\left(\chi_{\lambda}\right)$.

Definition 3.10. Let $\left(X, \tau_{1}, \tau_{2}, \tau_{3}, \tau_{4}\right)$ be a fuzzy q-topological space. The intersection of all fuzzy q-pre closed sets of $X$ containing a fuzzy subset $\chi_{\lambda}$ of $X$ is called fuzzy q-pre closure of $\chi_{\lambda}$ and is denoted by $\operatorname{Fqpcl}\left(\chi_{\lambda}\right)$.

Theorem 3.11. $\chi_{\lambda}$ is fuzzy q-semi open if and only if $\chi_{\lambda}=F q \operatorname{sint}\left(\chi_{\lambda}\right)$. 
Fuzzy Semi-Open Sets and Fuzzy Pre-Open Sets in Fuzzy Quad Topological Space Theorem 3.12. $F q \operatorname{sint}\left(\chi_{\lambda} \vee \chi_{\delta}>F q \operatorname{sint}\left(\chi_{\lambda}\right) \vee F q \operatorname{sint}\left(\chi_{\delta}\right)\right.$.

Theorem 3.13. $\chi_{\lambda}$ is a fuzzy q-semi closed set if and only if $\chi_{\lambda}=F q s c l\left(\chi_{\lambda}\right)$.

Theorem 3.14. Let $\chi_{\lambda}$ and $\chi_{\delta}$ be two fuzzy subsets of $\left(X, \tau_{1}, \tau_{2}, \tau_{3}, \tau_{4}\right)$ and $\chi_{\{x\}} \leq \tilde{1}_{X}$

a) $\chi_{\lambda}$ is fuzzy q-pre closed if and only if $\chi_{\lambda}=F q p c l\left(\chi_{\lambda}\right)$

b) If $\chi_{\lambda} \leq \chi_{\delta}$, then $\operatorname{Fqpcl}\left(\chi_{\lambda}\right) \prec \operatorname{Fqpcl}\left(\chi_{\delta}\right)$

c) $\chi_{\{x\}} \prec F q p c l\left(\chi_{\delta}\right)$ if and only if $\chi_{\lambda} \wedge \chi_{\delta} \neq \tilde{0}_{X}$ for every fuzzy q-pre-open set $f\left(\chi_{\lambda}\right)$ containing $f\left(\chi_{\{x\}}\right)$.

Theorem 3.15. Let $\chi_{\lambda}$ be a fuzzy subset of $\left(X, \tau_{1}, \tau_{2}, \tau_{3}, \tau_{4}\right)$, if there exist a fuzzy qpre-open set $\chi_{\delta}$ such that $\chi_{\lambda} \prec \chi_{\delta} \prec \operatorname{Fqcl}\left(\chi_{\lambda}\right)$, then $\chi_{\lambda}$ is fuzzy q-pre-open.

Theorem 3.16. In a fuzzy q-topological space $\left(X, \tau_{1}, \tau_{2}, \tau_{3}, \tau_{4}\right)$ the union of any two fuzzy q-semi-open sets is always a fuzzy q-semi-open set.

Proof: Let $\chi_{\lambda}$ and $\chi_{\delta}$ be any two fuzzy q-semi-open sets in $X$. Now

$$
\begin{gathered}
\chi_{\lambda} \vee \chi_{\delta} \leq F q c l\left(F q i n t \chi_{\lambda}\right) \vee F q c l\left(F q i n t \chi_{\delta}\right) \\
\Rightarrow \chi_{\lambda} \vee \chi_{\delta} \leq F q c l\left(F q i n t\left(\chi_{\lambda} \vee \chi_{\delta}\right)\right)
\end{gathered}
$$

Hence, $\left(\chi_{\lambda} \vee \chi_{\delta}\right.$ fuzzy q-semi-open sets.

Remark 3.17. The intersection of any two fuzzy q-semi-open sets may not be fuzzy qsemi-open sets as show in the following example

Example 3.18. Let $X=\{a, b, c, d\}$ be a non-empty fuzzy set.

Consider four fuzzy topologies on $\mathrm{X}$

$$
\begin{aligned}
\tau_{1} & =\left\{\tilde{1} X, \tilde{0} X, \chi_{\{a\}}\right\}, \\
\tau_{2} & =\left\{\tilde{1} X, \tilde{0} X, \chi_{\{a, d\}}\right\}, \\
\tau_{3} & =\left\{\tilde{1} X, \tilde{0} X, \chi_{\{c, d\}}\right\}, \\
\tau_{4} & =\left\{\tilde{1} X, \tilde{0} X, \chi_{\{a, c, d\}}\right\} .
\end{aligned}
$$

Fuzzy open sets in fuzzy q-topological spaces are union of all four fuzzy topologies. Then Fuzzy q-open sets of 


$$
F q S O(X)=\left\{\tilde{1} X, \tilde{0} X, \chi_{\{a\}}, \chi_{\{a, d\}}, \chi_{\{c, d\}}, \chi_{\{a, c, d\}}\right\} .
$$

Fuzzy q-semi-open set of $X$ is denoted by

Here

$$
F q S O(X)=\left\{\tilde{1} X, \tilde{0} X, \chi_{\{a\}}, \chi_{\{a, d\}}, \chi_{\{c, d\}}, \chi_{\{a, c, d\}}\right\}
$$

$\chi_{\{a, d\}} \wedge \chi_{\{c, d\}}=\chi_{\{d\}}>F q S O(X)$.

Theorem 3.19. If $\chi_{\lambda}$ is fuzzy q-open sets then $\chi_{\lambda}$ is fuzzy q-semi-open set.

Proof: Let $\chi_{\lambda}$ is a fuzzy q-open set.

Therefore $\chi_{\lambda}=F q i n t\left(\chi_{\lambda}\right)$

Now $\chi_{\lambda} \prec \operatorname{Fqcl}\left(\chi_{\lambda}\right)=\operatorname{Fqcl}\left(\operatorname{Fqint}\left(\chi_{\lambda}\right)\right)$ hence $\chi_{\lambda}$ is fuzzy q-semi-open set.

Theorem 3.20. Let $\chi_{\lambda}$ and $\chi_{\delta}$ be two fuzzy subsets of $X$ such that $\chi_{\delta} \prec \chi_{\lambda} \prec \operatorname{Fqcl}\left(\chi_{\delta}\right)$. If $\chi_{\delta}$ is a fuzzy q-semi-open set then $\chi_{\lambda}$ is also fuzzy q-semi-open set.

Proof: Given $\chi_{\delta}$ is fuzzy q-semi-open set.

So, we have $\chi_{\delta} \leq F q c l\left(F q i n t\left(\chi_{\delta}\right)\right) \leq F q c l\left(F q i n t\left(\chi_{\lambda}\right)\right)$.

Thus $\operatorname{Fqcl}\left(\chi_{\delta}\right) \leq \operatorname{Fqcl}\left(\operatorname{Fqint}\left(\chi_{\lambda}\right)\right)$ hence $\chi_{\lambda}$ is also fuzzy q-semi-open set.

\section{Fuzzy q-semi-continuity and fuzzy q-pre-continuity in fuzzy q-topological space}

Definition 4.1. A fuzzy function $\mathrm{f}$ from a fuzzy q-topological space $\left(X, \tau_{1}, \tau_{2}, \tau_{3}, \tau_{4}\right)$ into another fuzzy q-topological space $\left(\mathrm{Y}, \tau_{1}^{\prime}, \tau_{2}^{\prime}, \tau_{3}^{\prime}, \tau_{4}^{\prime}\right)$ is called fuzzy q-semicontinuous if $f^{-1}\left(\chi_{\lambda}\right)$ is fuzzy q-semi-open set in $X$ for each fuzzy q-open set $\chi_{\lambda}$ in Y.

Example 4.2. Let $X=\{a, b, c, d\}$ be a non-empty fuzzy set.

Consider four fuzzy topologies on $\mathrm{X}$

$$
\begin{aligned}
\tau_{1} & =\left\{\tilde{1} X, \tilde{0} X, \chi_{\{a\}}\right\}, \\
\tau_{2} & =\left\{\tilde{1} X, \tilde{0} X, \chi_{\{a, d\}}\right\}, \\
\tau_{3} & =\left\{\tilde{1} X, \tilde{0} X, \chi_{\{c, d\}}\right\}, \\
\tau_{4} & =\left\{\tilde{1} X, \tilde{0} X, \chi_{\{a, c, d\}}\right\} .
\end{aligned}
$$

Fuzzy open sets in fuzzy q-topological spaces are union of all four fuzzy topologies. Then fuzzy q-open sets of

$$
X=\left\{\tilde{1} X, \tilde{0} X, \chi_{\{a\}}, \chi_{\{a, d\}}, \chi_{\{c, d\}}, \chi_{\{a, c, d\}}\right\}
$$

Fuzzy q-semi-open set of $X$ is denoted by

$$
F q S O(X)=X=\left\{\tilde{1} X, \tilde{0} X, \chi_{\{a\}}, \chi_{\{a, d\}}, \chi_{\{c, d\}}, \chi_{\{a, c, d\}}\right\} .
$$


Fuzzy Semi-Open Sets and Fuzzy Pre-Open Sets in Fuzzy Quad Topological Space

Let $Y=\{1,2,3,4\}$ be a non-empty fuzzy set.

Consider four fuzzy topologies on $\mathrm{Y}$

$$
\begin{aligned}
& \tau_{1}^{\prime}=\left\{\tilde{1} Y, \tilde{0} Y, \chi_{\{1,4\}}\right\}, \quad \tau_{2}^{\prime}=\left\{\tilde{1} Y, \tilde{0} Y, \chi_{\{4\}}\right\}, \\
& \tau_{3}^{\prime}=\left\{\tilde{1} Y, \tilde{0} Y, \chi_{\{1,2\}}\right\}, \quad \tau_{4}^{\prime}=\left\{\tilde{1} Y, \tilde{0} Y, \chi_{\{1,2,4\}}\right\} .
\end{aligned}
$$

Fuzzy q-open sets of

$$
Y=\left\{\tilde{1} Y, \tilde{0} Y, \chi_{\{4\}}, \chi_{\{1,2\}}, \chi_{\{1,4\}}, \chi_{\{1,2,4\}}\right\} .
$$

Fuzzy q-semi-open set of $Y$ is

$$
F q S O(Y)=\left\{\tilde{1} Y, \tilde{0} Y, \chi_{\{4\}}, \chi_{\{1,2\}}, \chi_{\{1,4\}}, \chi_{\{1,2,4\}}\right\}
$$

Consider the fuzzy function $f: I^{x} \rightarrow I^{Y}$ is defined as

$$
\begin{gathered}
f^{-1}\left(\chi_{\{4\}}\right)=\chi_{\{a\}}, \quad f^{-1}\left(\chi_{\{1,2\}}\right)=\chi_{\{c, d\}}, \quad f^{-1}\left(\chi_{\{1,4\}}\right)=\chi_{\{a, d\}}, \\
f^{-1}\left(\chi_{\{1,2,4\}}\right)=\chi_{\{a, c, d\}}, f^{-1}\left(\tilde{0}_{Y}\right)=\left(\tilde{0}_{X}\right), f^{-1}\left(\tilde{1}_{Y}\right)=\left(\tilde{1}_{x}\right) .
\end{gathered}
$$

Since the inverse image of each fuzzy q-open set in $Y$ under $f$ is fuzzy q-semi-open set in $X$. Hence $f$ is fuzzy q-semi-continuous function.

Definition 4.2. A fuzzy function $\mathrm{f}$ defined from a fuzzy $\mathrm{q}$-topological space $\left(X, \tau_{1}, \tau_{2}, \tau_{3}, \tau_{4}\right)$ into another fuzzy q-topological space $\left(\mathrm{Y}, \tau_{1}^{\prime}, \tau_{2}^{\prime}, \tau_{3}^{\prime}, \tau_{4}^{\prime}\right)$ is called fuzzy q-pre-continuous function if $f^{-1}\left(\chi_{\lambda}\right)$ is fuzzy q-pre-open set in $X$ for each fuzzy q-open set $\chi_{\lambda}$ in $Y$.

Example 4.3. Let $X=\{a, b, c, d\}$ be a non-empty fuzzy set.

Consider four fuzzy topologies on $\mathrm{X}$

$$
\begin{array}{ll}
\tau_{1}=\left\{\tilde{1} X, \tilde{0} X, \chi_{\{a\}}\right\}, & \tau_{2}=\left\{\tilde{1} X, \tilde{0} X, \chi_{\{a, d\}}\right\}, \\
\tau_{3}=\left\{\tilde{1} X, \tilde{0} X, \chi_{\{b, d\}}\right\}, & \tau_{4}=\left\{\tilde{1} X, \tilde{0} X, \chi_{\{a, b, d\}}\right\} .
\end{array}
$$

Fuzzy open-sets in fuzzy q-topological spaces are union of all four fuzzy topologies. Then fuzzy q-open sets of 


$$
X=\left\{\tilde{1} X, \tilde{0} X, \chi_{\{a\}}, \chi_{\{a, d\}}, \chi_{\{b, d\}}, \chi_{\{a, b, d\}}\right\}
$$

Fuzzy q-pre-open set of $X$ is denoted by

$$
F q P O(X)=\left\{\tilde{1} X, \tilde{0} X, \chi_{\{a\}}, \chi_{\{a, d\}}, \chi_{\{b, d\}}, \chi_{\{a, b, d\}}\right\} .
$$

Let $Y=\{1,2,3,4\}$ be a non-empty fuzzy set.

Consider four fuzzy topologies on $\mathrm{Y}$

$$
\begin{aligned}
& \tau_{1}^{\prime}=\left\{\tilde{1} Y, \tilde{0} Y, \chi_{\{1,4\}}\right\}, \quad \tau_{2}^{\prime}=\left\{\tilde{1} Y, \tilde{0} Y, \chi_{\{4\}}\right\}, \\
& \tau_{3}^{\prime}=\left\{\tilde{1} Y, \tilde{0} Y, \chi_{\{1,2\}}\right\}, \quad \tau_{4}^{\prime}=\left\{\tilde{1} Y, \tilde{0} Y, \chi_{\{1,2,4\}}\right\} .
\end{aligned}
$$

Fuzzy q-open sets of $Y=\left\{\tilde{1} Y, \tilde{0} Y, \chi_{\{4\}}, \chi_{\{1,2\}}, \chi_{\{1,4\}}, \chi_{\{1,2,4\}}\right\}$.

Fuzzy q-pre-open set of $Y$ is denoted by

$$
F q P O(Y)=\left\{\tilde{1} Y, \tilde{0} Y, \chi_{\{4\}}, \chi_{\{1,2\}}, \chi_{\{1,4\}}, \chi_{\{1,2,4\}}\right\} .
$$

Consider the fuzzy function $f: I^{x} \rightarrow I^{Y}$ is defined as

$$
\begin{gathered}
f^{-1}\left(\chi_{\{4\}}\right)=\chi_{\{a\}}, \quad f^{-1}\left(\chi_{\{1,2\}}\right)=\chi_{\{b, d\}}, \quad f^{-1}\left(\chi_{\{1,4\}}\right)=\chi_{\{a, d\}}, \\
f^{-1}\left(\chi_{\{1,2,4\}}\right)=\chi_{\{a, b, d\}}, f^{-1}\left(\tilde{0}_{Y}\right)=\left(\tilde{0}_{X}\right), f^{-1}\left(\tilde{1}_{Y}\right)=\left(\tilde{1}_{x}\right) .
\end{gathered}
$$

Since the inverse image of each fuzzy q-open set in $Y$ under $f$ is fuzzy q-pre-open set in $X$. Hence $f$ is fuzzy q-pre-continuous function.

Theorem 4.4. Let $f:\left(X, \tau_{1}, \tau_{2}, \tau_{3}, \tau_{4}\right) \rightarrow\left(Y, \tau_{1}^{\prime}, \tau_{2}^{\prime}, \tau_{3}^{\prime}, \tau_{4}^{\prime}\right)$ be a fuzzy q-pre-continuous open function .If $\chi_{\lambda}$ is a fuzzy q-pre-open set of $X$, then $f\left(\chi_{\lambda}\right)$ is a fuzzy q-pre-open set in $Y$.

Proof: First, let $\chi_{\lambda}$ be fuzzy q-pre-open set in $X$.There exist a fuzzy q-open set $\chi_{\delta}$ in $X$ such that $\chi_{\lambda} \prec \chi_{\delta} \prec F q c l\left(\chi_{\lambda}\right)$. Since $\mathrm{f}$ is a fuzzy q-open function then $f\left(\chi_{\delta}\right)$ is a fuzzy $\mathrm{q}$-open set in $Y$. Since $\mathrm{f}$ is a fuzzy q-continuous function, we have

$$
f\left(\chi_{\lambda}\right) \prec f\left(\chi_{\delta}\right) \prec f\left(\operatorname{Fqcl}\left(\chi_{\lambda}\right)\right) \prec \operatorname{Fqcl}\left(f\left(\chi_{\lambda}\right)\right) .
$$

This show that $f\left(\chi_{\lambda}\right)$ is fuzzy q-pre-open in $Y$.

Let $\chi_{\lambda}$ be a fuzzy q-pre-open in $X$.There exist a fuzzy q-pre-open set $\chi_{\delta}$ such that

$$
\chi_{\delta} \prec \chi_{\lambda} \prec \operatorname{Fqcl}\left(\chi_{\delta}\right) .
$$

Since $f$ is a fuzzy q-continuous function, we have by the proof of first part, $f\left(\chi_{\delta}\right)$ is a fuzzy q-pre-open in $X$. Therefore $f\left(\chi_{\lambda}\right)$ is a fuzzy q-pre-open in $Y$. 
Fuzzy Semi-Open Sets and Fuzzy Pre-Open Sets in Fuzzy Quad Topological Space

Theorem 4.5. Let $f:\left(X, \tau_{1}, \tau_{2}, \tau_{3}, \tau_{4}\right) \rightarrow\left(Y, \tau_{1}^{\prime}, \tau_{2}^{\prime}, \tau_{3}^{\prime}, \tau_{4}^{\prime}\right)$ be a fuzzy q-pre continuous open function .If $\chi_{\lambda}$ is a fuzzy q-pre-open set of $Y$, then $f^{-1}\left(\chi_{\lambda}\right)$ is a fuzzy q-pre-open in $X$.

Proof: First, let $\chi_{\lambda}$ be a fuzzy q-pre-open set of $Y$.There exist a fuzzy q-open set $\chi_{\delta}$ in $Y$. Such that $\chi_{\lambda} \prec \chi_{\delta} \prec F q c l\left(\chi_{\lambda}\right)$. Since $f$ is a fuzzy q-open, we have

$$
f^{-1}\left(\chi_{\lambda}\right) \prec f^{-1}\left(\chi_{\delta}\right) \prec f^{-1}\left(\operatorname{Fqcl}\left(\chi_{\lambda}\right)\right) \prec \operatorname{Fqcl}\left(f^{-1}\left(\chi_{\lambda}\right)\right) .
$$

Since $f$ is a fuzzy q-pre continuous function, $f^{-1}\left(\chi_{\delta}\right)$ is a fuzzy q-pre-open set in $X$. By theorem $3.13, f^{-1}\left(\chi_{\lambda}\right)$ is a fuzzy q-pre-open set in $X$.

Theorem 4.6. The following are equivalent for a function $f:\left(X, \tau_{1}, \tau_{2}, \tau_{3}, \tau_{4}\right) \rightarrow$ $\left(Y, \tau_{1}^{\prime}, \tau_{2}^{\prime}, \tau_{3}^{\prime}, \tau_{4}^{\prime}\right)$

a) $f$ is a fuzzy q-pre continuous function;

b) The inverse image of each fuzzy q-closed set of $Y$ is fuzzy q-pre closed in $X$;

c) $F q p c l\left(f^{-1}\left(\chi_{\lambda}\right)\right) \prec f^{-1}\left(F q p c l\left(\left(\chi_{\lambda}\right)\right)\right.$ for every subset $\chi_{\lambda}$ of $Y$.

d) $f\left(F q p c l\left(\chi_{\delta}\right)\right) \prec F q c l\left(f\left(\chi_{\delta}\right)\right)$ for every subset $\chi_{\delta}$ of $X$.

Theorem 4.7. If $f:\left(X, \tau_{1}, \tau_{2}, \tau_{3}, \tau_{4}\right) \rightarrow\left(Y, \tau_{1}^{\prime}, \tau_{2}^{\prime}, \tau_{3}^{\prime}, \tau_{4}^{\prime}\right)$ and

$$
g:\left(Y, \tau_{1}^{\prime}, \tau_{2}^{\prime}, \tau_{3}^{\prime}, \tau_{4}^{\prime}\right) \rightarrow\left(Z, \tau_{1}^{\prime \prime}, \tau_{2}^{\prime \prime}, \tau_{3}^{\prime \prime}, \tau_{4}^{\prime \prime}\right)
$$

be two fuzzy q-semi continuous function then

$$
\text { fog: }\left(X, \tau_{1}, \tau_{2}, \tau_{3}, \tau_{4}\right) \rightarrow\left(Z, \tau_{1}^{\prime \prime}, \tau_{2}^{\prime \prime}, \tau_{3}^{\prime \prime}, \tau_{4}^{\prime \prime}\right)
$$

may not be fuzzy q-semi continuous function .

Theorem 4.8. Every fuzzy q-continuous function is a fuzzy q-semi continuous function.

Theorem 4.9. Let $f:\left(X, \tau_{1}, \tau_{2}, \tau_{3}, \tau_{4}\right) \rightarrow\left(Y, \tau_{1}^{\prime}, \tau_{2}^{\prime}, \tau_{3}^{\prime}, \tau_{4}^{\prime}\right)$ be bijective. Then the following conditions are equivalent:

i) $f$ is a fuzzy q-semi-open continuous function.

ii) $f$ is a fuzzy q-semi closed continuous function and

iii) $f^{-1}$ is a fuzzy q-semi continuous function.

Proof: (i) $\rightarrow$ (ii) Suppose $\chi_{\lambda}$ is a fuzzy q-closed set in $X$. Then $\tilde{1}_{X}-\chi_{\lambda}$ is a

Fuzzy q-open set in $X$. Now by (i) $f\left(\tilde{1}_{X}-\chi_{\lambda}\right)$ is a fuzzy q-semi-open set in $Y$. Now since $f^{-1}$ is fuzzy bijective function so $f\left(\tilde{1}_{X}-\chi_{\lambda}\right)=Y-f\left(\chi_{\lambda}\right)$. Hence $f\left(\chi_{\lambda}\right)$ is a fuzzy q-semi closed set in $Y$. Therefore $f$ is a fuzzy q-semi closed continuous function. 
(ii) $\rightarrow$ (iii) Let $f$ is a fuzzy q-semi closed map and $\chi_{\lambda}$ be a fuzzy q-closed set of $X$. Since $f^{-1}$ is bijective so $\left(f^{-1}\right)^{-1} \chi_{\lambda}$ which is a fuzzy q-semi-closed set in $Y$. Hence $f^{-1}$ is a fuzzy q-semi continuous function.

(iii) $\rightarrow$ (i) Let $\chi_{\lambda}$ be a fuzzy q-open set in $X$. Since $f^{-1}$ is a fuzzy q-semi continuous function so $\left(f^{-1}\right)^{-1} \chi_{\lambda}=f\left(\chi_{\lambda}\right)$ is a fuzzy q-semi open set in $Y$. Hence $f$ is fuzzy qsemi-open continuous function.

Theorem 4.10. Let $X$ and $Y$ are two fuzzy q-topological spaces. Then $f:\left(X, \tau_{1}, \tau_{2}, \tau_{3}, \tau_{4}\right) \rightarrow\left(Y, \tau_{1}^{\prime}, \tau_{2}^{\prime}, \tau_{3}^{\prime}, \tau_{4}^{\prime}\right)$ is fuzzy q-semi-continuous function if one of the followings holds:

i) $f^{-1}\left(F q \sin t \chi_{\lambda}\right) \leq F q \operatorname{sint}\left(f^{-1}\left(\chi_{\lambda}\right)\right.$, for every fuzzy q-open set $\chi_{\lambda}$ in $Y$.

ii) $F q \operatorname{scl}\left(f^{-1}\left(\chi_{\lambda}\right)\right) \leq f^{-1}\left(F q \operatorname{sint}\left(\chi_{\lambda}\right)\right)$, for every fuzzy q-open set $\chi_{\lambda}$ in $Y$.

Proof: Let $\chi_{\lambda}$ be any fuzzy $q$-open set in $Y$ and if condition (i) is satisfied then

$$
f^{-1}\left(F q \sin t \chi_{\lambda}\right) \leq F q \operatorname{sint}\left(f^{-1}\left(\chi_{\lambda}\right)\right.
$$

We get $f^{-1}\left(\chi_{\lambda}\right) \leq F q \operatorname{sint}\left(f^{-1}\left(\chi_{\lambda}\right)\right.$.

Therefore $f^{-1}\left(\chi_{\lambda}\right)$ is a fuzzy q-semi-open set in $X$. Hence $f$ is a fuzzy q-semi-continuous function. Similarly we can prove (ii).

Theorem 4.11. A function $f:\left(X, \tau_{1}, \tau_{2}, \tau_{3}, \tau_{4}\right) \rightarrow\left(Y, \tau_{1}^{\prime}, \tau_{2}^{\prime}, \tau_{3}^{\prime}, \tau_{4}^{\prime}\right)$ is called fuzzy q-semi open continuous function if and only if

$$
f\left(F q \operatorname{sint}\left(\chi_{\lambda}\right)\right) \leq F q \operatorname{sint}\left(f\left(\chi_{\lambda}\right)\right)
$$

for every quad open set $\chi_{\lambda}$ in $X$.

Proof: Suppose that $f$ is a quad semi open continuous function.

Since $F q \operatorname{sint}\left(f\left(\chi_{\lambda}\right) \leq \chi_{\lambda}\right.$ so $f\left(F q \operatorname{sint}\left(f\left(\chi_{\lambda}\right)\right) \leq f\left(\chi_{\lambda}\right)\right.$.

By hypothesis $F q \operatorname{sint}\left(f\left(\chi_{\lambda}\right)\right)$ is a fuzzy q-semi-open set and $F q \operatorname{sint}\left(f\left(\chi_{\lambda}\right)\right)$ is largest fuzzy q-semi-open set contained in $f\left(\chi_{\lambda}\right)$ so $f\left(F q \operatorname{sint}\left(\chi_{\lambda}\right)\right) \leq F q \operatorname{sint}\left(f\left(\chi_{\lambda}\right)\right)$.

Conversely, suppose $\chi_{\lambda}$ is a fuzzy q-open set in $X$.So $f\left(F q \operatorname{sint}\left(\chi_{\lambda}\right)\right) \leq F q \operatorname{sint}\left(f\left(\chi_{\lambda}\right)\right)$.

Now since $\chi_{\lambda}=F \operatorname{sint}\left(\chi_{\lambda}\right)$ so $f\left(\chi_{\lambda}\right) \leq F q \operatorname{sint}\left(f\left(\chi_{\lambda}\right)\right)$

Therefore $f\left(\chi_{\lambda}\right)$ is a fuzzy q-semi-open set in $Y$ and $f$ is a fuzzy q-semi-open continuous function.

Theorem 4.12. A function $f:\left(X, \tau_{1}, \tau_{2}, \tau_{3}, \tau_{4}\right) \rightarrow\left(Y, \tau_{1}^{\prime}, \tau_{2}^{\prime}, \tau_{3}^{\prime}, \tau_{4}^{\prime}\right)$ is called fuzzy q-semi closed continuous function if and only if $f\left(F q s c l\left(\chi_{\lambda}\right)\right) \leq F q \operatorname{scl}\left(f\left(\chi_{\lambda}\right)\right)$ for every fuzzy q-closed set $\chi_{\lambda}$ in $X$. 
Fuzzy Semi-Open Sets and Fuzzy Pre-Open Sets in Fuzzy Quad Topological Space

Proof: Suppose that $f$ is a fuzzy q-semi closed continuous function. Since

$\chi_{\lambda} \leq F q \operatorname{scl}\left(\chi_{\lambda}\right)$ so $f\left(\chi_{\lambda}\right) \leq f\left(F q s c l\left(\chi_{\lambda}\right)\right)$. By hypothesis, $f\left(F q \operatorname{scl}\left(\chi_{\lambda}\right)\right)$, is a fuzzy q-semi closed set and $f\left(F q \operatorname{scl}\left(\chi_{\lambda}\right)\right)$ is smallest fuzzy q-semi closed set containing $f\left(\chi_{\lambda}\right)$ so $f\left(F q s c l\left(\chi_{\lambda}\right)\right) \leq F q s c l\left(f\left(\chi_{\lambda}\right)\right)$.

Conversely, suppose $\chi_{\lambda}$ is a fuzzy q-closed set in $X$. So $f\left(F q s c l\left(\chi_{\lambda}\right)\right) \leq F q \operatorname{Fcl}\left(f\left(\chi_{\lambda}\right)\right)$.

Since $\chi_{\lambda}=F q \operatorname{Fcl}\left(\chi_{\lambda}\right)$ so $F q \operatorname{scl}\left(f\left(\chi_{\lambda}\right)\right) \leq f\left(\chi_{\lambda}\right)$.

Therefore $f\left(\chi_{\lambda}\right)$ is a fuzzy q-semi closed set in $Y$ and $f$ is fuzzy q-semi closed continuous function.

Theorem 4.13. Every fuzzy q-semi continuous function is fuzzy q-continuous function.

\section{Conclusion}

In this paper the idea of fuzzy q-semi-open sets, fuzzy q-pre-open sets, fuzzy qcontinuous function, fuzzy q-semi continuous function and fuzzy q-pre continuous function in fuzzy q-topological spaces were introduced and studied.

\section{REFERENCES}

1. A.Kandil, Biproximities and fuzzy bitopological spaces, Simon Steven, 63 (1989) 4566.

2. A.S.Bin Sahana, On fuzzy strongly semi-continuity and fuzzy pre continuity, Fuzzy Sets and Systems, 44 (1991) 303-308.

3. A.S.Mashhour, M.E.El-Monsef Abd and S.N.El. Deep, On pre continuous and weak pre continuous mappings, Proc. Math. Phys. Soc. Egypt, 53 (1981) 47-53.

4. C.L.Change, Fuzzy topological spaces, J. Math. Anal. Appl., 24 (1968) 182-190.

5. D.V.Mukundan, Introduction to quad topological spaces, Int. Journal of Scientific and Eng. Research, 4(7) (2013) 2483-2485.

6. K.K.Azad, On fuzzy semi-continuity, fuzzy almost continuity and fuzzy weakly continuity, J. Math. Anal. Appl., 82 (1981) 14-32.

7. L.A.Zadeh, Fuzzy sets, Information and Control, 8 (1965) 338-353.

8. M.Jelic, A decomposition of pair wise continuity, J. Inst. Math. Comp. Sci. Math. Ser, 3 (1990) 25-29.

9. M. Kovar, On 3-topological version of thet-reularity, Internat. J. Matj, Sci., 23(6) (2000) 393-398.

10. N.F.Hammed and Yahya Abid Mohammed, Certain types of separation axioms in tri topological spaces. Iraqi Journal of Science, 52 (2) (2011) 212-217.

11. N.Levine, Semi open sets and semi-continuity in topological spaces, Amer. Math., 70 (1963) 36- 41. 
12. R.Sharma, B.A.Deole and S.Verma, Fuzzy semi-open sets and fuzzy pre-open sets in fuzzy tri topological space, International Journal of Mathematics Trends and Technology, 55(6) (2018) 420-429.

13. S.Kumar, On fuzzy pair wise $\alpha$-continuous and fuzzy pair wise pre-continuity, Fuzzy Sets and Systems, 62 (1994) 231-238.

14. S.N.Maheshwari and R.Prasad, Semi-open sets and semi-continuous functions in bitopological spaces, Math. Notae., 26 (1977) 29-37.

15. S.Palaniammal, Study of tri-topological spaces, Ph. D. Thesis, (2011).

16. S.S.Thakur and R.Malviya, Semi-open sets, Semi continuity and semi open mapping in fuzzy bitopological Spaces, Fuzzy Sets and Systems, 64 (1994) 421-426.

17. U.D.Tapi and R.Sharma and B.A.Deole, Semi-open sets and Pre-open sets in Tri Topological space, Journal on Mathematics, 5(3) (2016) 41-47.

18. U.D.Tapi and R.Sharma and B.A.Deole, Semi-open sets and pre-open sets in quad topological space, International Journal of Computer \& Mathematical Sciences, 6(9) (2017) 15-20. 